\title{
Faint blue galaxies revisited
}

\author{
Henry C. Ferguson \\ Space Telescope Science Institute, 3700 San Martin Drive, Baltimore, MD 21218, USA \\ email: ferguson@stsci.edu
}

\begin{abstract}
If dwarf-elliptical galaxies formed their stars very rapdily (on timescales of less than 1 Gyr), they may in principle be detectable out to high redshift. Prior to the discovery of cosmic acceleration, it appeared that rapid and late formation dwarf elliptical galaxies might be required to explain the number counts of faint galaxies. A plausible hypothesis emerged: that photoionization by the UV background prevents gas cooling in low-mass halos until $z \lesssim 1.5$. The discovery of cosmic acceleration eased the tension between predicted galaxy number counts and galaxy-evolution models. Nevertheless, there is some evidence for relatively late star formation in nearby dE's, and the photoionization delay mechanism still appears to have some merit. It is thus of interest to look back in time to see if we can find starbursting dwarf galaxies at moderate redshift. We review the connection between faint-blue galaxies and bursting-dwarf galaxies and discuss some attempts to identify progenitors to dE galaxies in the Hubble Ultra Deep Field (HUDF) observations. We find roughly 85 galaxies in the HUDF with redshifts $0.6<z<1.1$ that appear to have formed most of their stars at $z<1.5$. Of these, $70 \%$ have half-light radii less than $1.5 \mathrm{kpc}$. These are thus "smoking gun" candidates for dwarf galaxies that are either collapsing for the first time at moderate redshifts or have otherwise been unable to form stars for more than $1 / 3$ of a Hubble time.
\end{abstract}

Keywords. galaxies: dwarf, galaxies: evolution

\section{Introduction}

A variety of interesting cosmological issues converge when we consider the evolution of dwarf elliptical galaxies. There are some strong tensions between observations and theory, and some serious unknowns in the physical processes that might be shaping these galaxies. This of course makes the topic interesting and provides fodder for excellent conferences in beautiful places!

Among the various tensions, a few are relevant to studies of distant galaxies because they determine how many we faint galaxies should expect to see and how bright they should be at high redshift.

(a) The dE galaxies that we obseve in the local universe show a luminosity function $\phi(L) d L$ continues to rise to the limits of our ability to detect galaxies (Trentham, Sampson \& Banerji 2005). However, the slope of this function is significantly shallower than the predicted slope of the mass-function of dark-matter halos (e.g. Moore et al. 1999). Either something has supressed star formation in low-mass halos, or the halo mass function itself deviates from the predictions. This missing-dwarf problem is one aspect of the "substructure crisis" that currently challenges Cold-Dark Matter (CDM) theory.

(b) Certain observations favor rapid star-formation in $\mathrm{dE}$ galaxies. In particular, the reasonably well-defined metallicity-luminosity relation (summarized by Dekel \& Woo 2003 ) is easiest to explain if star formation in dE galaxies was truncated by supernovadriven winds. Such winds are efficient if the star-formation episode is rapid enough (a few $\times 10^{7} \mathrm{yr}$ ) to allow overlap of expanding supernova remnants (Dekel \& Silk 1986). However, recent observations suggest that local-group dSph galaxies have $\alpha /$ Fe element 
ratios indicative of significant enrichment by type Ia supernovae (e.g. Venn et al. 2004). The characteristic timescale for enrichment by type Ia SNe is generally thought to be $\sim 1$ Gyr. Furthermore, evidence for rotational support and/or cold gas in some dE's suggests that star formation was not rapid and may not have run to completion (e.g. Geha et al. 2003; Conselice et al. 2003; van Zee et al. 2004; Michielsen et al. 2004).

(c) One possible solution to the missing dwarf problem is that star-formation in lowmass halos has been "squelched" due to photoionization by the metagalactic UV background (Efstathiou 1992; Babul \& Rees 1992; Babul \& Ferguson 1996; Somerville 2002). Photoionization prevents cooling of $\mathrm{HI}$ and the subsequent $\mathrm{H}_{2}$ formation needed to form stars. The UV background becomes important at redshifts $6<z<20$, and drops siginficantly from $z=1$ to the present (Scott et al. 2002). A possible result is that at least some low-mass halos retain their gas, but do not form many stars until 4-7 Gyr after reionization. Unfortunately, while the theory seems well founded, there is very little observational evidence to support it. The star-formation histories of local-group dSph galaxies do not suggest a preferred epoch to their formation either prior to reionization or at low redshifts. Stellar population indicators for cluster dE's show more diversity than expected if such galaxies formed all their stars before reionization, but do not show a clear indication of a long, coherent hiatus in star formation. Dwarf irregular galaxies of course do show evidence for recent star formation, but it is difficult to determine star-forming histories over the timespan 6-12 Gyr in the past.

In summary, while $\mathrm{dE}$ (and $\mathrm{dSph}$ ) galaxies appear to be the most common type of galaxy in the local universe, there do not appear to be enough of them. Those that we see have some well-defined systematic trends, suggestive of some common mechanism that regulates star formation and feedback. But there is considerable diversity in ages and evidence increasingly disfavors models where the star formation occured in short bursts.

Deep surveys may help sort things out by allow us to detect and study the progenitors of today's dE galaxies at significant lookback times.

\subsection{A brief history of faint-blue galaxies}

The idea of testing world models by counting galaxies goes back to the early part of the 20th century. Counting galaxies became an industry in the late 1970s to late 1990s as bigger telescopes, better detectors, and increasingly sophisticated measurement techniques provided large gains in sensitivity (Kron 1978; Tyson 1988; Koo \& Kron 1992; Ellis 1997). These surveys revealed that the number counts of galaxies were in excess of expectations from prevailing cosmological models (with $\Omega_{M}=\Omega_{\text {tot }}=1$ ), and that this excess was most pronounced at blue wavelengths (Yoshii \& Takahara 1988; Guiderdoni \& Rocca-Volmerange 1991). The redshift distribution was relatively broad and peaked at redshifts $z<1$ (Cowie et al. 1991; Crampton et al. 1995). The faint-blue galaxies did not appear to be strongly clustered (Efstathiou 1995).

The steep CDM halo mass function presented a possible solution. If star-formation in low-mass halos were delayed until $z<1$ by the UV background, and if stars subsequently formed on timescales $\sim 10^{7} \mathrm{yr}$, then these small dwarfs could in principle dominate the faint-blue galaxy number counts (Efstathiou 1992; Babul \& Rees 1992). However in the $\Omega_{m}=1$ SCDM model, Ferguson \& Babul (1998) showed that these dwarfs would fade too slowly to be consistent with the counts and colors of galaxies in the Hubble Deep Field. Thus, while late formation of $\mathrm{dE}$ galaxies had the potential to solve the faint-blue galaxy problem, the extreme model required to reconcile SCDM with the number counts did not appear to work without some additional tweaking (e.g. adopting a steep IMF).

The current $\Lambda \mathrm{CDM}$ concordance cosmological model with $\Omega_{\mathrm{tot}}, \Omega_{m}, \Omega_{\Lambda}=1.0,0.3,0.7$ has both more volume and more time at high redshifts than the previously-favored model. 

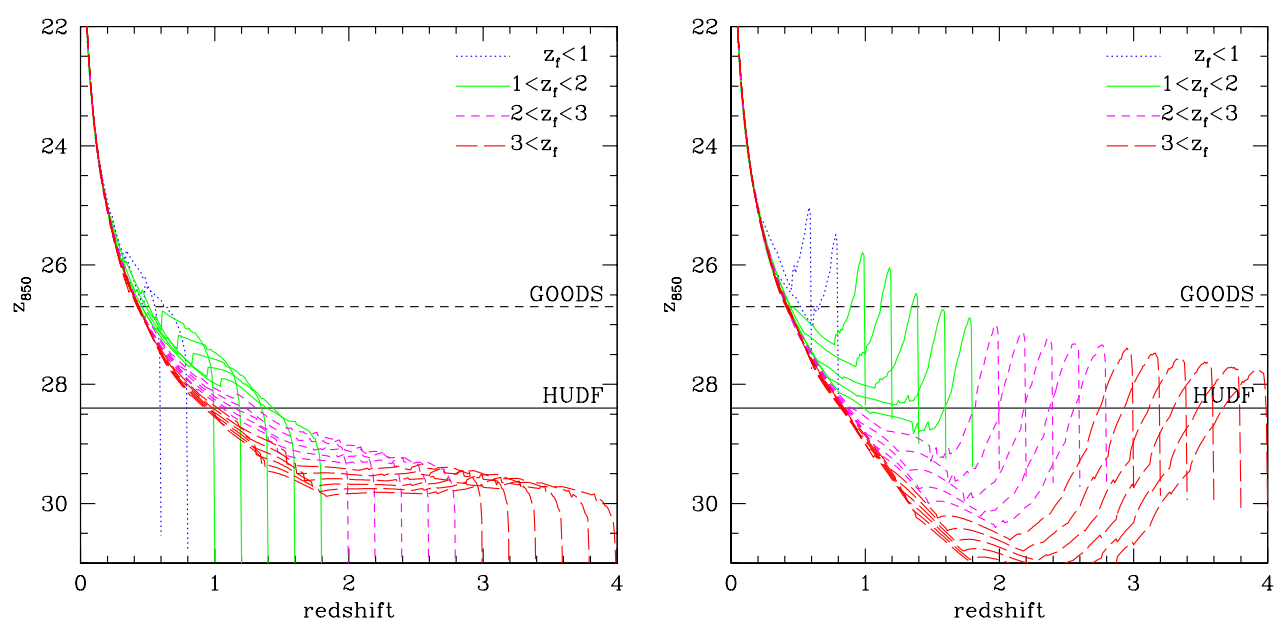

Figure 1. (Left) Apparent HST $z_{850}$ magnitude as a function of redshift for $\mathrm{dE}$ galaxies forming at a variety of redshifts with an exponential star-formation timescale $\tau_{\mathrm{SF}}=10^{9} \mathrm{yr}$. The dashed line shows the magnitude limit for GOODS for a 0.2 square arcsecond aperture. The solid line shows the detection limit for the HUDF. (Right). Same for galaxies with $\tau_{\mathrm{SF}}=10^{8} \mathrm{yr}$.

Galaxy counts are therefore not nearly as interesting as they used to be. Few papers have been published in the past five years comparing modern $\Lambda$ CDM hierarchical models to galaxy counts. Nagashima et al. (2002) show a comparison to one set of models indicating good, but not perfect, agreement.

\subsection{The visibility of $d E$ galaxies}

Consider a dE galaxy with a present-day absolute magnitude $M_{B}=-14$. The roughly $10^{9} M_{\odot}$ of stars could have formed within the $\sim 10^{7} \mathrm{yr}$ timescale of supernova driven winds, in which case it might have had a peak star-formation rate of $100 M_{\odot} \mathrm{yr}^{-1}$, comparable to typical Lyman-break galaxies seen at redshift $z \sim 3$. Alternatively, the galaxy might form stars on the $10^{8}$ to $10^{9}$ yr timescales predicted by models with a gentler form of self regulation (Lin \& Murray 1992; Hensler et al. 2004). In Fig. 1 we show apparent magnitude vs. redshift for dwarf galaxies forming at a variety of redshifts with starformation timescales of $10^{9} \mathrm{yr}$ (left) and $10^{8} \mathrm{yr}$ (right), all of which end up at $M_{B}=-14$ at the present epoch. The HST Advanced Camera for Surveys (ACS) z-band magnitude limits are shown for Great Observatories Origins Deep Survey (GOODS) and the Hubble Ultra-Deep Field (HUDF). Independent of the star-forming history, a dE galaxy would be visible in GOODS to $z \sim 0.4$ and in the HUDF to $z \sim 0.8$. If the star-formation timescale is longer than $10^{9} \mathrm{yr}$, even the HUDF would not be deep enough to see the formation phase of this hypothetical $\mathrm{dE}$ if it takes place at $z>1.5$. On the other hand, if the star-formation timescale is $10^{8} \mathrm{yr}$, the HUDF is deep enough to see the formation phase out to $z>4$.

\subsection{Emission-line searches bursting dwarfs}

The magnitude limit of unbiased redshift surveys with Keck and the VLT is $R \sim 24$, which is too bright for our proto-typical dE galaxy at $z>0.5$ even with star-formation timescales as short as $10^{7} \mathrm{yr}$. We must rely on emission lines and/or photometric redshifts to find these galaxies and constrain their redshifts. For redshifts $z>0.4, \mathrm{H} \alpha$ line becomes increasingly difficult to detect from the ground through the forest of night-sky lines. The HST NICMOS grism survey found about $0.5 \mathrm{H} \alpha$ emitters per square arc minute with 
typical star-formation rates $\sim 20 M_{\odot} \mathrm{yr}^{-1}$ in the redshift range $0.7<z<1.9$ (McCarthy et al. 1999). In visual appearance, at least some of these galaxies look compact enough to be dwarfs. What we do not see in this survey is a pileup of emission line galaxies at $z<1$, which might have signaled a renewed epoch of dwarf-galaxy formation.

The [OII] 3727 ̊ line, while a less directly correlated with star-formation, is accessible in the optical out to $z \sim 1$.4. Lotz, Martin \& Ferguson (2003) carried out an [OII] emissionline search for star-forming galaxies in two clusters at $z \sim 0.4$. Interestingly, one cluster, A851, showed a factor of 3-4 excess of emission-line objects relative to the field, while the other cluster MS1512.4+3647, showed no excess. The detection limits were sufficient to detect galaxies with star-formation rates as low as $0.13 M_{\odot} \mathrm{yr}^{-1}$. Many of fainter emissionline galaxies detected in these clusters are either in the field, or infalling into the clusters. While they could be progenitors of present-day $\mathrm{dE}$ galaxies, it is unlikely that such accretion of dwarfs since $z=0.4$ could produce the numbers or spatial distribution of dwarfs seen locally in places like the Virgo and Fornax Clusters. Thus, we are probably seeing at $z=0.4$ a snapshot of an ongoing process.

Lilly, Carollo \& Stockton (2003) used the $\mathrm{R}_{23}$ method to measure metallicities of galaxies in the redshift range $0.5<z<0.9$ with magnitudes $I_{A B}<22.5$. They found that $75 \%$ of the galaxies had metallicities comparable to nearby galaxies of similar luminosities. Of the remaining 25\%, only few appeared have the sizes consistent with being dwarf galaxies. The implication is that $\mathrm{dE}$ galaxies probably formed stars on moderately long timescales $\left(>10^{8} \mathrm{yr}\right)$ or before $z=1$.

\subsection{Dwarfs in the Hubble Ultra Deep Field}

For most galaxies fainter than $R \sim 24$, we must rely on indirect techniques to estimate redshifts. Photometric redshift estimates typically involve fitting spectral templates to galaxy broad-band photometry. The most important feature that is shifting through the optical and near-IR bandpasses out to redshifts $z \sim 2$ is the Balmer or $4000 \AA$ break. Instead of fitting templates, we can define photometric quantities (differences of colors) that identify the spectral inflection around this break. This "spectral inflection selection" technique is akin to the Lyman-break technique in that careful selection of colors can isolate star-forming galaxies in well-defined redshift intervals. The detailed selection criteria involve multiple bands and would take too much space to describe here. As an example, if we define three HST colors as $c_{1}=B_{435}-V_{606}$ and $c_{2}=V_{606}-i_{775}$, and $c_{3}=i_{775}-z_{850}$, then a good selection criterion for galaxies at $z \sim 0.8$ is to require $-0.7<c_{1}-c_{2}<0.1$ and $0<c_{2}-c_{3}<0.7$. Figure 2 (left panel) shows the redshift selection functions for three different redshift intervals derived from simulations. The righthand panel shows that there is reasonable agreement with the spectroscopic redshift distribution for GOODS galaxies that meet the selection criteria.

Sersic profiles $-I(r) \propto \exp \left[\left(-r / r_{e}\right)^{1 / n}\right]$ - have been fit to the HUDF images (Ravindranath, private communication). A large fraction of galaxies in our photometrically identified redshift slices are well fit by profiles that are nearly exponential $(n<1.5)$. While this is reminiscent of local dE galaxies, disk galaxies also have $n \sim 1$, and both inspection of the images and axial-ratio measurements indicate that at least a small fraction of our sample has disk-like morphologies.

Table 1 shows the absolute magnitude limits and approximate number densities. The volume estimates are very rough, and are formally model dependent because the redshift selection-function depends on the spectral-energy distributions of the evolving $\mathrm{dE}$ galaxies. Nevertheless, these densities are low enough that it appears unlikely that a large fraction of the $\mathrm{dE}$ population formed most of their stars in any of these redshift intervals. Consider two extreme hypotheses: (a) most $\mathrm{dE}$ galaxies form most of their stars above 

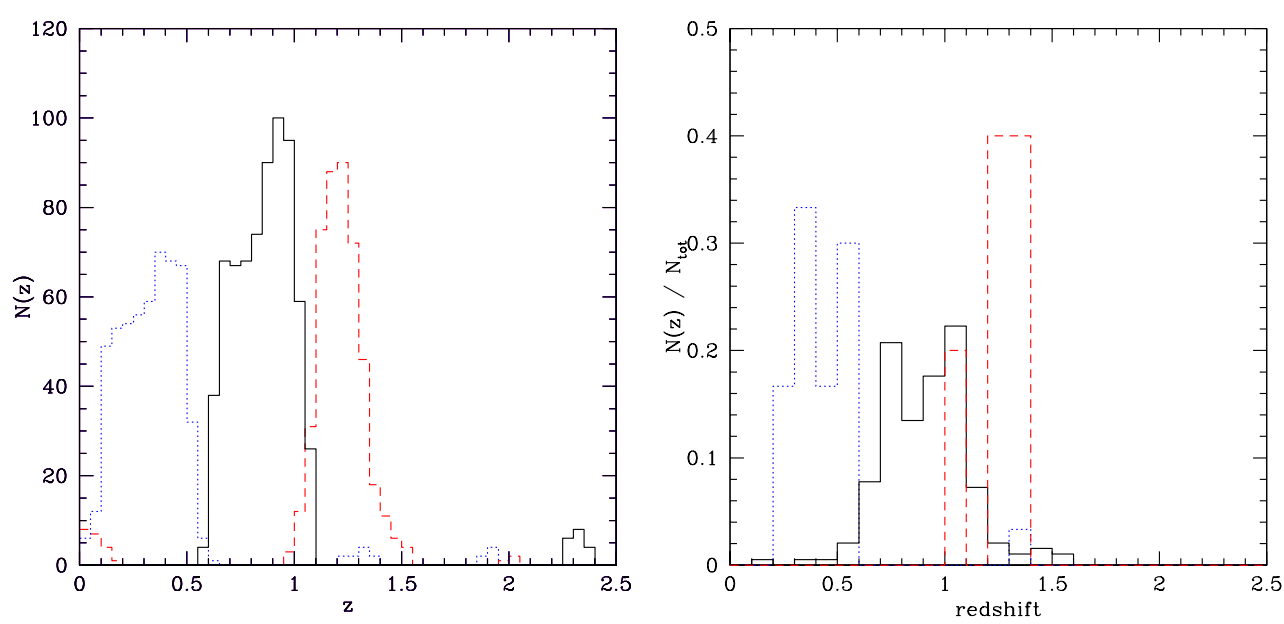

Figure 2. (Left) Redshift selection functions for searching for moderate-redshift dE galaxies. The spectral-inflection selection is efficient at identifying star-forming galaxies or galaxies with strong $4000 \AA$ breaks. It selects against galaxies that are either outside the redshift intervals of interest, or highly reddened. The selection functions shown here were derived by estimating colors for a wide variety of SED models. (Right) Measured redshift distribution for galaxies in the GOODS CDF-S field that meet the same selection criteria.

Table 1. HUDF redshift slices

\begin{tabular}{lcccc}
\hline $\begin{array}{l}\text { Redshift } \\
\text { range }\end{array}$ & $\begin{array}{c}\text { mag limit } \\
\left(i_{775}\right)\end{array}$ & $\begin{array}{c}M_{B} \text { (rest) } \\
\text { limit }\end{array}$ & $\mathrm{N}$ & $\begin{array}{c}\text { Density } \\
\left(\times 10^{-3} \mathrm{Mpc}^{-} 3\right)\end{array}$ \\
\hline $0-0.5$ & 27 & -15.2 & 4 & 4.4 \\
$0.6-1.0$ & 27.5 & -16.2 & 144 & 21 \\
$1.0-1.3$ & 27 & -18.3 & 20 & 2.7
\end{tabular}

$z=3$ on short timescales and fade to the present day, and (b) most dE galaxies form their stars at $z<1.2$ with star-formation timescales $\tau_{\mathrm{SF}} \sim 1$ Gyr. To estimate how many $\mathrm{dE}$ we should expect to see in these two scenarios, we need to adopt a local luminosity function (and force both scenarios to match it). The local dE LF in the field is not well constrained. We adopt the Blanton et al. (2005) LF here in order to have some plausible numbers to work with. (Strictly speaking, their LF is for all dwarfs, not just dE.) Using this LF, in case (a) we should be able to detect to $0.01 L^{*}$ in the redshift interval $0.6<z<1$. Integrating from $0.01 L^{*}$ to $0.18 L^{*}$, we expect roughly 200 dwarfs in this redshift range in the HUDF. However, the SED's in case (a) are sufficiently smooth that these galaxies would generally not fulfill the selection criteria that we have adopted. On the other had, if the galaxies formed at $z \sim 1.2$, they would meet the selection criteria, and the galaxies would be about 2.2 mag brighter at $z=1$ than in case (a). Thus number densities would increase dramatically because we probe further down the intrinsic mass function. Integrating from $0.0016 L^{*}$ to $0.025 L^{*}$ we would predict roughly 600 galaxies in the HUDF, meeting our selection critiera for $0.6<z<1.1$. This is significantly higher than the number of galaxies that we find in the HUDF (144) or the number (69) that have half-light radii less than $1.5 \mathrm{kpc}$, and hence are likely to be dwarfs (see Fig. 3).

It is interesting to examine the colors of these faint, moderate-redshift galaxies. An example color-color diagram is shown in Fig. 4 . This shows $B_{435}-V_{606}$ vs. $z_{850}-H_{160}$ for the sample of galaxies selected by spectral inflection to lie in the redshift range 


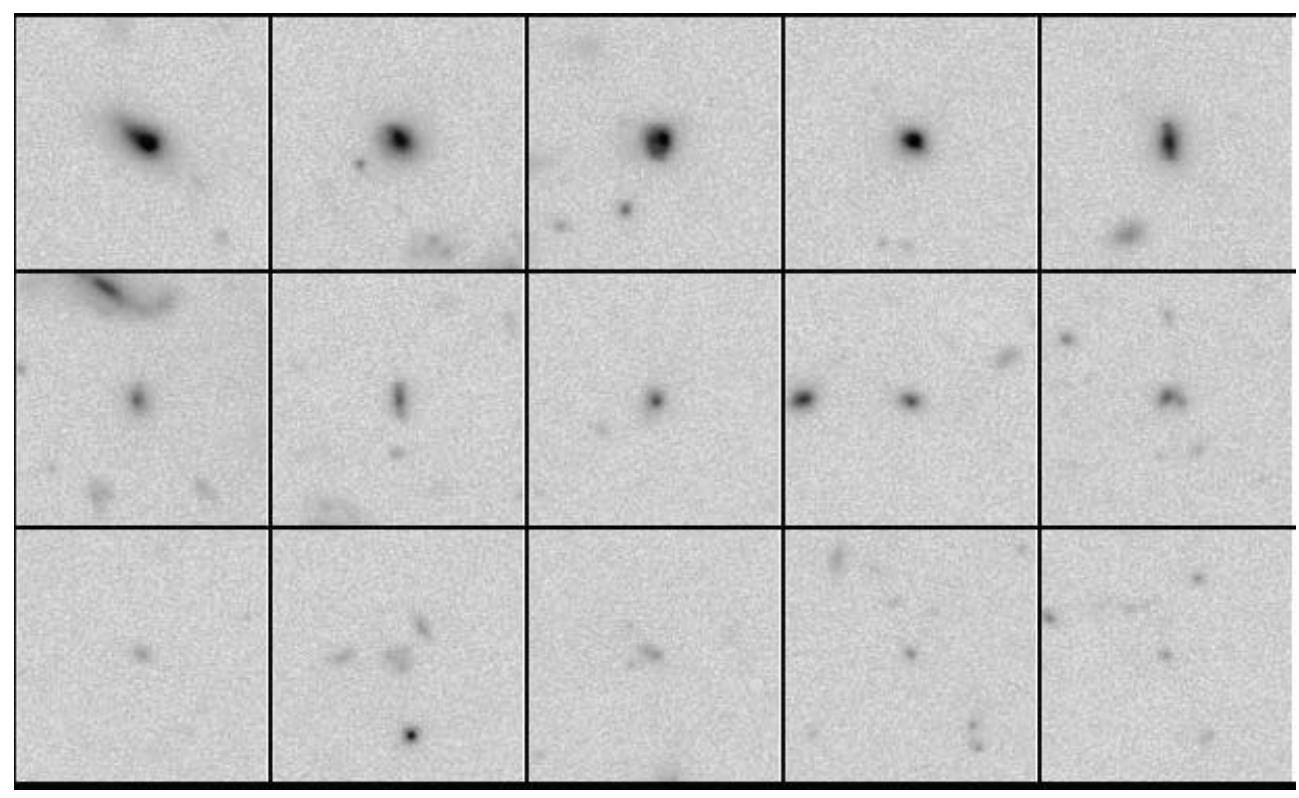

Figure 3. Montage of galaxies selected via spectral inflection to lie in the redshift range $0.6<z<1.1$. The candidates shown all have half-light radii $r_{h}<1.5 \mathrm{kpc}$ and we show in the top panel the brightest five candidates, in the middle panel some candidates near the midpoint in luminosity, and the bottom panel the five faintest candidates. While some of the galaxies have disk-like morphologies, many are reminiscent of $\mathrm{dE}$ galaxies. Most have exponential surface-brightness profiles. The galaxy at the top left has a star-formation rate of approximately $1.4 M_{\odot} \mathrm{yr}^{-1}$; the galaxy at the lower right has $S F R \sim 0.03 M_{\odot} \mathrm{yr}^{-1}$.

$0.6<z<1.1$. We have computed colors for a large suite of models, spanning formation redshifts $0.1-5$ in steps $\Delta z=0.1$ metallicity $[\mathrm{Fe} / \mathrm{H}]=-1.3$, burst durations $\log \left(\tau_{S F}\right)=$ $6,7,8,9,10.5$ and Calzetti et al. (2000) extinctions $E(B-V)=0.2,0.4$. In the left panel we show all models that began forming stars at redshift $z<1.5$. In the right panel we show all models that began forming stars at $z>1.5$. There is a clear separation of the models, and it is evident that over half of the galaxies appear to be incompatible with redshifts of formation $z_{f}>1.5$. To be precise, 84 out of 148 galaxies in this redshift interval lie bluewards of $B_{435}-V_{606}=0.3$, a color which separates all models with $z_{f}>1.5$ from most models with $z_{f}<1.5$. Of these 84 galaxies, most $(70 \%)$ have halflight radii less than $1.5 \mathrm{kpc}$. A similar behavior is seen in the next higher redshift slice $1<z<1.3$, where we find an additional 22 young galaxies $\left(z_{f}<1.5\right)$ of which $60 \%$ have $r_{h}<1.5 \mathrm{kpc}$. While these are clearly interesting galaxies, the total population of bursting-dwarf candidates that we have identifed between $z=0.6$ and $z=1.3$ represents only $\sim 11 \%$ of the galaxy counts in the magnitude range $H_{606}=22-26$. We have no constraints on bursting dwarfs in our low-redshift slice $(z<0.5)$, because the high- $z_{f}$ and lower- $z_{f}$ occupy the same locus in this color-color diagram.

In summary, the number counts of faint-blue galaxies are not in serious conflict with $\Lambda C D M$ cosmological models. Nevertheless, there are interesting faint-blue galaxies in deep images, at least some of which may be progenitors of present-day dE galaxies. Spectral-inflection selection in the HUDF reveals in intriguing population of faint, compact galaxies that appear to have waited to $z<1.5$ to form stars. While these may be progenitors of some $\mathrm{dE}$ galaxies, if most $\mathrm{dE}$ galaxies formed their stars at $z<1.5$, we would have expected to find many more such candidates. 

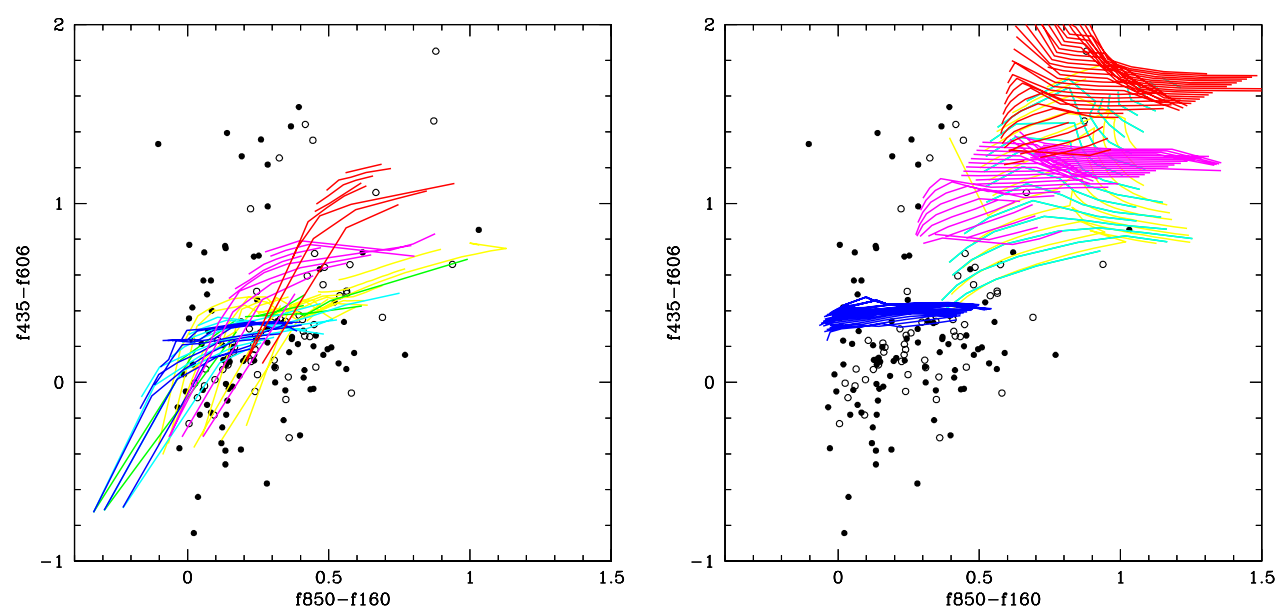

Figure 4. Observed colors of galaxies at $0.6<z<1.1$ compared to models. In both panels, the solid points are galaxies with $r_{h}<1.5 \mathrm{kpc}$, and the open circles are galaxies with larger half-light radii. All galaxies have been identified via spectral inflection, as described in the text. The left panel shows a suite of models (described in the text), all of which began forming stars at $z_{f}<1.5$. The models are viewed at a finely sampled set of redshifts $0<z<z_{f}$, but the colors are only plotted when the model spectral inflections meet the selection criteria used for the galaxies. The models with short $\tau_{S F}$ tend to congregate toward the lower right in this diagram. (For readers viewing these diagrams in color: yellow curves have $\log \tau_{S F}=6,7$, green has $\log \tau_{S F}=8$, cyan has $\log \tau_{S F}=9$, blue has $\log \tau_{S F}=10.5$, all with no dust; magenta has $\log \tau_{S F}=9$ and $E(B-V)=0.2$ and red has $\log \tau_{S F}=9$ and $E(B-V)=0.4$.) To explain the data points that lie below and to the right of most of the models may require lower metallicites, emission lines, or an unusual IMF. In the right panel, we show the suite of models with $z_{f}>1.5$. Even the bluest models in this set (those with nearly constant star formation over a Hubble time) are redder than more than half of the observed galaxies. This suggests that many of the galaxies in this sample are bursting dwarf galaxies that could evolve into present-day dE galaxies.

\section{Acknowledgements}

We would like to acknowledge the support and collaboration of the GOODS and HUDF teams in this endeavor. Thanks in particular to Swara Ravindranath for providing GALFIT results for the HUDF. Support for this work was provided by NASA through grant GO09583.01-96A from the Space Telescope Science Institute, which is operated by the AURA, Inc., under NASA contract NAS5-26555.

\section{References}

Babul, A. \& Rees, M.J. 1992, MNRAS 255, 346

Babul, A. \& Ferguson, H.C. 1996, ApJ 458, 100

Blanton, M., Lupton, R.H., Schlegel, D.J., Strauss, M.A. Brinchman, J., Fukugita, M. \& Loveday, J. 2005, ApJ submitted, astro-ph/0410164

Calzetti, D., Armus, L., Bohlin, R.C., Kinney, Al, Koorneef, J. \& Storchi-Bergmann, T. 2000 ApJ 533, 682

Conselice, C.J., O’Niel, K., Gallagher, J.S. \& Wyse, R.F.G. 2003 ApJ 591, 167

Cowie, L.L., Songaila, A. \& Hu, E.M. 1991 Nature 354, 460

Crampton, D., Le Fev́re, O., Lilly, S.J. \& Hammer, F. 1995 ApJ 455, 96

Dekel, A. \& Silk, J. 1986 ApJ 303, 39

Dekel, A. \& Woo, J. 2003 MNRAS 344, 1131

Efstathiou, G. 1992, MNRAS 256, 43 
Efstathiou, G. 1995, MNRAS 272, L25

Ellis, R.S. 1997, ARAA 35, 389

Ferguson, H.C. \& Babul, A. 1998, MNRAS 296, 585

Geha, M., Guhathakurta, P. \& van der Marel, R.P. 2003, AJ 126, 1794

Guiderdoni, B. \& Rocca-Volmerange, B. 1991 A $\& A$ 252, 435

Hensler, G., Theis, Ch. \& Gallager, J.S. III. $2004 A \& A$ 426, 25

Koo, D. \& Kron, R. 1992 ARAA 30, 613

Kron, R. 1978, Thesis, University of California at Berkeley

Lin, D.N. \& Murray, S.D. 1992 ApJ 394, 523

Lilly, S.J., Carollo, C.M. \& Stockton, A. 2003 ApJ 597, 730

Lotz, J.M., Martin, C.L. \& Ferguson, H.C. 2003 ApJ 596, 143

McCarthy, P.J., et al. 1999 ApJ 520, 548

Michielsen, D., de Rijcke, S., Zeilinger, W.W., Prugniel, P., Dejonghe, H. \& Roberts, S. 2004 MNRAS 353, 1293

Moore, B., et al. 1999 ApJ 524, L19

Nagashima, M., Yoshii, Y., Totani, T. \& Gouda, N. 2002, ApJ 578, 675

Scott, J., Bechtold, J., Morita, M., Dobrzycki, A. \& Kulkarni, V. P. 2002, ApJ 571, 665

Somerville, R.S. 2002, ApJ. 572, L23

Trentham, N., Sampson, L. \& Banerji, M. 2005, MNRAS 357, 783

Tyson, J. A. 1988, AJ 96, 1

van Zee, L., Skillman, E.D. \& Hanes, M.P. 2004, AJ 128, 121

Venn K.A., Irwin, M., Shetrone, M.D., Tout, C.A., Hill, V. \& Tolstoy, E. 2004, AJ 128, 1177

Yoshii Y. \& Takahara, F. 1988, ApJ 326, 1

\section{Discussion}

CONSELICE: What fraction of your dwarf sample is dE and dIrr?

FErguson: Good question. The visual impression is that most of the small galaxies look like late-type dwarfs or dEs, but we have not yet looked at the statistical distribution of concentration indices, Sersic parameters, or ellipticities.

ZAGGIA: Have you searched for any spatial clustering around possible galaxy parents?

FERGuson: not yet.

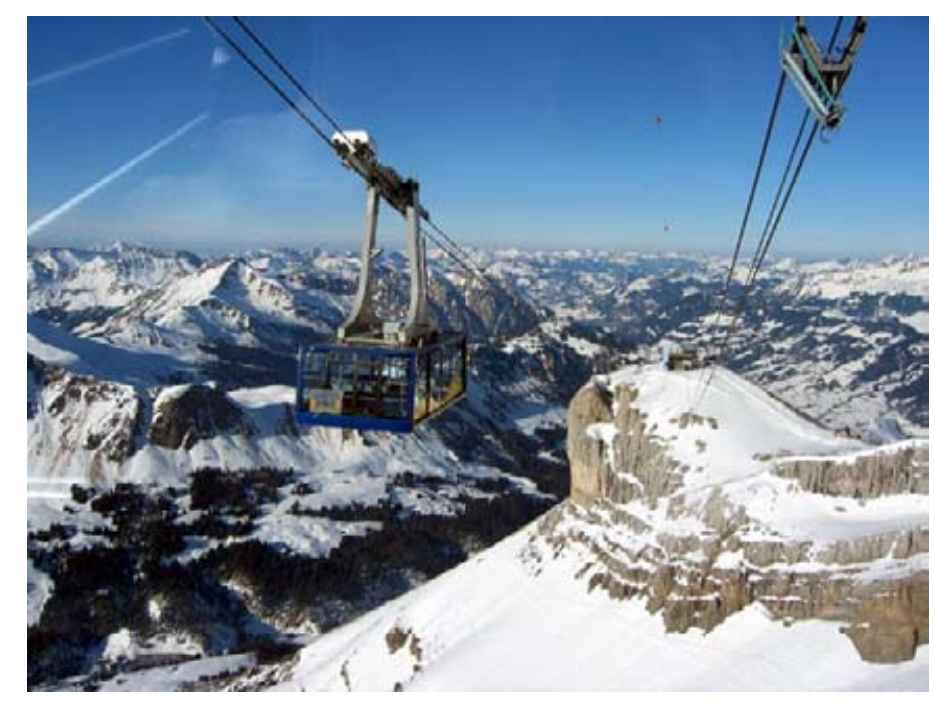

